\title{
BMJ Comprehensive abortion care: evidence OPen of improvements in hospital-level indicators in Tigray, Ethiopia
}

\author{
Ndola Prata, ${ }^{1}$ Suzanne Bell, ${ }^{1}$ Amanual Gessessew ${ }^{2}$
}

To cite: Prata N, Bell S, Gessessew A. Comprehensive abortion care: evidence of improvements in hospitallevel indicators in Tigray, Ethiopia. BMJ Open 2013;3: e002873. doi:10.1136/ bmjopen-2013-002873

- Prepublication history for this paper is available online. To view these files please visit the journal online (http://dx.doi.org/10.1136/ bmjopen-2013-002873).

Received 12 March 2013 Revised 26 April 2013 Accepted 30 May 2013

This final article is available for use under the terms of the Creative Commons Attribution Non-Commercial 3.0 Licence; see http://bmjopen.bmj.com

${ }^{1}$ UC Berkeley Bixby Center for Population, Health and Sustainability, School of Public Health, UC Berkeley, Berkeley, California, USA 2Department of Gynaecology and Obstetrics, Mekelle University College of Health Sciences, Mekelle, Tigray, Ethiopia

Correspondence to Dr Ndola Prata; ndola@berkeley.edu

\section{ABSTRACT}

Objective: Approximately $18 \%$ of maternal deaths in East Africa is attributable to unsafe abortion.

Availability of comprehensive abortion care (CAC) services at all levels of the healthcare system, including medical abortion, has the potential to increase access to safe abortion thereby reducing the burden of unsafe abortion. This study sought to assess trends in abortion-related morbidity indicators in referral hospitals.

Design: Researchers conducted a secondary data analysis on retrospectively collected data.

Methods: Data analysed were collected from four hospitals in the Tigray region of Ethiopia that took part in a CAC pilot project. Providers were trained in mid2009 to provide abortion services using all available technologies. Data records from hospitals were collected in 2012 for the years 2006 through 2012; $2006 / 2007$ data were too sparse to include in the analyses.

Results: Trends in abortion-related services show a significant decrease in treatment of incomplete abortion, inverting the relationship between safe terminations and treatment of incompletes as a percentage of total abortions. Medication abortion was nearly non-existent in 2008, but increased steadily, representing $80 \%$ of total procedures in 2012 . The inclusion of medication abortion and availability of CAC also contributed to a decline in inpatient procedures and prevalence of complications.

Conclusions: The trends observed in the data demonstrate how increased availability of CAC services at all levels of the healthcare system, among other factors, can contribute to reductions in the burden of unsafe abortion at referral hospitals.

\section{INTRODUCTION}

Unsafe abortion is a leading cause of maternal mortality and morbidity throughout the world. ${ }^{1}$ This is a particularly salient issue in sub-Saharan Africa, which has the highest maternal mortality ratio (MMR) of any region of the world-900/100000 livebirths. ${ }^{2}$ Estimates indicate that approximately $14 \%$ of maternal deaths in this part of the

\section{ARTICLE SUMMARY}

Article focus

- Availability of comprehensive abortion care (CAC) services at all levels of the healthcare system, including medical abortion, has the potential to increase access to safe abortion thereby reducing the burden of unsafe abortion.

- This study sought to assess trends in abortionrelated morbidity indicators in referral hospitals from 2008 to 2012.

\section{Key messages}

- Trends in abortion-related services show a significant decrease in treatment of incomplete abortion, inverting the relationship between safe terminations and treatment of incompletes as a percentage of total abortions.

- Medication abortion was nearly non-existent in 2008, but increased steadily and statistically significantly, representing $80 \%$ of total procedures in 2012.

- Availability of CAC services at all levels of the healthcare system, in particular the inclusion of medication methods, can increase access to safe abortion services, thereby reducing the financial, personnel, maternal and societal burden of unsafe abortion.

Strengths and limitations of this study

- This study contributes to the body of evidence that assesses tertiary-level abortion service indicators after the introduction of CAC at all levels of the healthcare system in Tigray, Ethiopia.

- Data quality and completeness was an important limitation, although we do not believe the results are biased as a result of this.

- Ideally, we would have been able to collect additional information regarding the percentage of total clients presenting at the hospital for abortion-related services from all obstetric services provided each year, as well as abortionrelated maternal mortality and referral data.

world is due to unsafe abortion. ${ }^{3}$ In East Africa specifically, maternal mortality attributable to unsafe abortion is $18 \%$, with an abortion maternal mortality ratio (AMMR) of $100 / 100000$ live-births-the highest in the 
world. ${ }^{3}$ These statistics are often associated with low rates of contraceptive use, high numbers of unwanted pregnancy and limited access to safe abortion and postabortion care (PAC). ${ }^{4}$ Data illustrate how unsafe abortion is consistently higher in places with restrictive abortion laws, reaffirming that women who experience unplanned pregnancies are highly likely to have an abortion regardless of the legal status in their country. ${ }^{5}$

In 2008, the annual abortion rate of Ethiopia was 23/1000 women between the ages 15 and $44 .{ }^{6}$ An estimated 382000 women received induced abortions that year, $52600(14 \%)$ of which were treated for complications in facilities, and only $103000(27 \%)$ of which were legal procedures in health facilities. ${ }^{6}$ Some evidence seems to indicate that AMMR in tertiary facilities has been trending downward, although the change is not statistically significant. ${ }^{7}$ Findings from this same study indicated that $70 \%$ of all abortions at the tertiary facility under investigation were conducted using sharp metallic curettage, which is not recommended by the WHO. ${ }^{7} 8$ Improving access to safe abortion using recommended procedures (ie, vacuum aspiration and medication) would decrease complications and financial and personnel costs.

Secondary and tertiary hospital indicators tend to capture a significant proportion of the burden of unsafe abortion when other lower level facilities do not provide abortion services on a large scale. ${ }^{9-11}$ For example, one hospital in Tigray reported $13 \%$ of all hospital admissions and $61 \%$ of gynaecological admissions were abortionrelated from 2002 to $2004 .{ }^{11}$ On a national level, direct expenditures (drugs/supplies, personnel, transport/hospitalisation/overtime fees) related to treating patients requiring PAC in 2008 amounted to approximately $\$ 7.6$ million, the average cost per treated patient being $\$ 31 .^{12}$ Incorporating indirect costs (overhead and capital costs) the conservative estimate for cost of PAC in 2008 was $\$ 11$ million. Furthermore, personnel spend on average from $101 \mathrm{~min}$ to conduct an uncomplicated incomplete abortion to up to $22 \mathrm{~h}$ to treat a uterine perforation; these estimates include total time spent on treating or attending to patients, summed across all types of providers that attend to a given patient. ${ }^{12}$

Current evidence illustrates the effectiveness of misoprostol for abortion-related services. Misoprostol can be used alone for pregnancy termination with its effectiveness ranging from $75 \%$ to $90 \%$ depending on dose, route and gestational age. ${ }^{813-15}$ For treatment of incomplete abortion and miscarriage, misoprostol achieves similar effectiveness as manual vacuum aspiration (MVA) ${ }^{16-19}$ Additionally, a recent study demonstrated that mid-level providers can provide medication abortion (MA) with similar safety and effectiveness as doctors. ${ }^{20}$ This supports previous evidence that mid-level providers should be trained to perform MA, especially when considering that they tend to serve more rural/low-income populations, thus allowing for improved access among marginalised women. ${ }^{21} 22$
Given recent changes in technology availability (medication and surgical) in addition to increased access to abortion care at peripheral health facilities, this study sought to assess trends in abortion-related service indicators in referral hospitals in Tigray, Ethiopia.

\section{Background}

In 2005, Ethiopia took steps to reduce the burden of unsafe abortion and its contribution to maternal mortality (the 2011 estimate of the MMR is 676/100 000 livebirths) by making changes to their penal code. ${ }^{23} 24$ According to the law, termination of pregnancy is not punishable where: (1) the pregnancy is the result of rape or incest; (2) the continuance of the pregnancy endangers the life of the mother or the child; (3) where the child has an incurable and serious deformity or (4) where the pregnant woman is physically as well as mentally unfit to bring up the child. ${ }^{24}$ It is also allowed 'in the case of grave and imminent danger which can be averted only by an immediate intervention'. ${ }^{24}$ These changes to the legal status of abortion, along with efforts to increase knowledge of this change and the capacity of providers to perform safe abortions and treatment of incomplete abortions, has the potential to greatly reduce maternal morbidity and mortality attributable to unsafe abortion.

In 2006, the Ministry of Health adapted the technical guidelines and provided direction to help providers and facilities implement the newly revised law in hospitals and health centres. ${ }^{25}$ In total, there are 16 hospitals, 235 health centres and 600 health posts that are currently functional in Tigray. The Tigray Regional Health Bureau (TRHB) and Ipas began working in August 2006 and ultimately trained providers from 50 of these public facilities (38 health centres and 12 hospitals) to provide comprehensive abortion care (CAC) and monitored the availability, utilisation, and quality of safe abortion care (SAC) services. $^{26}$ The efforts sought to improve abortion-related services in public sector facilities. ${ }^{26}$

From May 2009 to December 2010, Venture Strategies Innovations, the University of California, Berkeley Bixby Center for Population, Health and Sustainability and Mekelle University collaborated with the TRHB on a pilot project that aimed to increase access to CAC services at all levels of the healthcare system by introducing medication methods from health posts to hospitals. The simplicity of misoprostol only abortion allows abortion-related services to take place at health posts, the lowest level facility in the healthcare system, thus broadening access points for abortion care in the most rural areas. This project involved four hospitals, nine health centres and 20 health posts (all public facilities). Health posts $(n=20)$, staffed by Health Extension workers (HEWs), could treat women with incomplete abortions and miscarriages up to 12 weeks gestation with misoprostol and could terminate pregnancies with misoprostol up to 9 weeks gestation. Health centres $(n=9)$ provided terminations and treatment of incomplete abortion up to 12 weeks gestation. 
Hospitals ( $n=4)$ served as referral centres for complicated cases and services needed during the second trimester. In most instances, within and outside the context of this project, health posts refer patients to health centres that provide the necessary abortion care and health centres refer patients to hospitals for any services they are unable to provide. Women received misoprostol only for safe terminations in health posts prior to 9 weeks gestation; other facilities could use misoprostol only for treatment of incomplete abortions and mifepristone-misoprostol regimens for pregnancy terminations. Women took the misoprostol orally and received additional misoprostol in instances of incomplete termination after MA.

The primary objective of the project was to demonstrate that CAC services can safely and effectively be provided at all levels of the healthcare system using both medication and surgical methods, thus maximising human and infrastructure capacity. ${ }^{27}$

\section{METHODS}

A data extraction form was used to collect data from the abortion logbooks in the four Tigray hospitals that participated in the CAC pilot project; these hospitals had also previously been involved in the Ipas trainings on SAC, which included MVA. Variables of interest included total number of abortion-related services provided, type of abortion (induced abortion, treatment of incomplete abortion, other), method of treatment used (dilation and curettage (D\&C), MVA, MA, other), level of complications (serious, minor, none) and inpatient versus outpatient treatment. Inpatient treatment indicates that the patient was admitted to the hospital and occupied a hospital bed for some amount of time; outpatient indicates it was an ambulatory care procedure. Data extracted correspond to the period September 2006 until May 2012. Data from 2006 to 2007 were too sparse to include in the analyses, thus the data analysed were from 2008 to 2012.

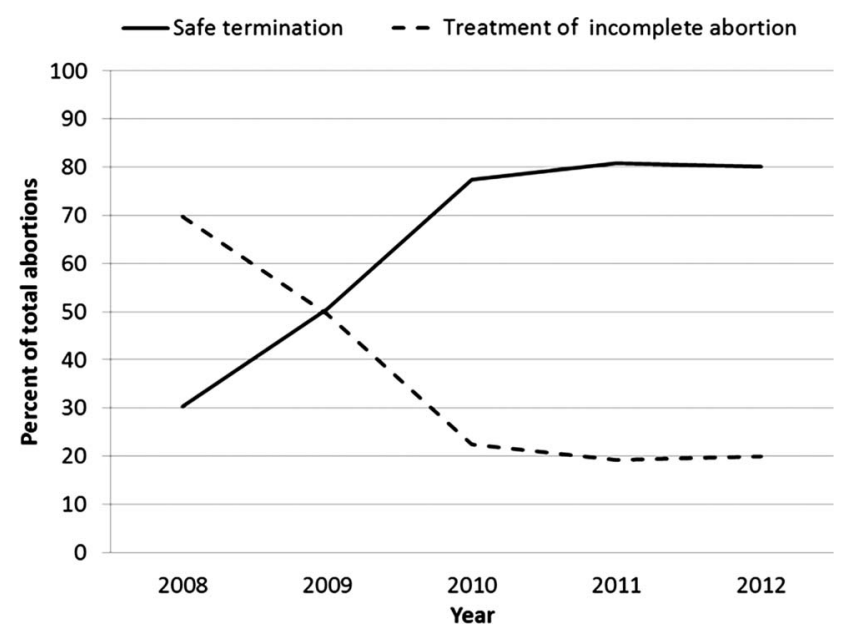

Figure 1 Trends of abortion-related services provided in four tertiary hospitals in Tigray, Ethiopia (2008-2012).
Proportions for each of the indicators were calculated and then plotted using Excel. To determine whether changes in proportions between years were statistically significant, we used a two-tailed $\mathrm{z}$ test for comparison of two proportions. The confidence level was set at $\mathrm{p}=0.05$. All statistical analyses were carried out using STATA V.11.0. ${ }^{28}$

\section{RESULTS}

Trends in abortion-related indicators show significant changes over the time period under investigation. In 2008, a total of 644 abortion-related services were recorded, which increased to 881, 2289, 3053 and 2152 in 2009, 2010, 2011 and the first half of 2012, respectively. As seen in figure 1, the relationship between safe terminations and treatment of incomplete abortions inverted between 2008 and 2012. In 2008, 70\% of abortions services provided in the studied referral hospitals were treatment of incomplete abortion (figure 1). By 2010, $78 \%$ were safe terminations (figure 1). Changes in proportions between years were highly significant up until 2010 and slightly less significant from 2010 to 2011 (table 1). The difference in proportions from 2011 to 2012 was not significant; proportions levelled off at this point (table 1).

In 2008, the vast majority (62\%) of abortion-related services utilised MVA; other surgical methods like D\&C were the second most common type of treatment (36\%; figure 2). Alternatively, almost no treatment was performed using MA (0.3\%; figure 2). The proportion of MA increased significantly the following 2 years ( $\mathrm{p}<0.001)$, most prominently between 2009 and 2010, and it remained high through 2012 as surgical methods decreased (table 1). By 2012, 78\% of abortion-related services utilised medication methods (figure 2).

Figure 3 illustrates the changes in the severity of complications over time. In 2008, $5 \%$ of women who presented at the participating hospitals for abortion-related services presented with serious complications, whereas $48 \%$ and $47 \%$ experienced no or minor complications, respectively (figure 2). The proportion of women experiencing no complications trended upward significantly, as the proportion who experienced minor or serious complications trended downwards, significantly at times (figure 3 and table 1).

Figure 4 provides some information regarding the burden of abortion-related services on the hospitals. In 2008, 28\% of abortion-related services were inpatient procedures. This proportion increased slightly before decreasing significantly in 2010 and then increasing significantly in 2012. By 2012, 24\% of abortion-related services were inpatient procedures, down only slightly from 2008.

\section{DISCUSSION AND CONCLUSIONS}

Hospital-level data from this study illustrate significant improvements in abortion-related indicators from 2008 
Table 1 Changes in proportions of abortion-related service indicators and level of significance over time

\begin{tabular}{|c|c|c|c|c|c|c|c|c|}
\hline & \multicolumn{2}{|l|}{ 2008-2009 } & \multicolumn{2}{|l|}{ 2009-2010 } & \multicolumn{2}{|l|}{ 2010-2011 } & \multicolumn{2}{|l|}{ 2011-2012 } \\
\hline & Difference & p Value & Difference & p Value & Difference & p Value & Difference & p Value \\
\hline \multicolumn{9}{|l|}{ Type of abortion } \\
\hline Safe termination & +20.3 & $<0.001$ & +26.6 & $<0.001$ & +3.4 & 0.002 & -0.8 & 0.475 \\
\hline $\begin{array}{l}\text { Treatment of incomplete } \\
\text { abortion }\end{array}$ & -20.3 & $<0.001$ & -26.9 & $<0.001$ & -3.3 & 0.003 & +0.7 & 0.530 \\
\hline \multicolumn{9}{|l|}{ Type of treatment } \\
\hline Surgical & -17.9 & $<0.001$ & -12.7 & $<0.001$ & -0.7 & 0.271 & -0.6 & 0.331 \\
\hline MVA & -1.4 & 0.576 & -44.7 & $<0.001$ & +3.3 & 0.002 & +0.5 & 0.662 \\
\hline Medication & +18.8 & $<0.001$ & +58.1 & $<0.001$ & -2.5 & 0.037 & +2.2 & 0.072 \\
\hline Other & +0.6 & 0.149 & -0.6 & 0.026 & -0.2 & 0.099 & +0.4 & 0.007 \\
\hline \multicolumn{9}{|l|}{ Level of complications } \\
\hline None & +5.5 & 0.025 & +15.4 & $<0.001$ & +6.3 & $<0.001$ & +8.2 & $<0.001$ \\
\hline Minor & -6.8 & 0.005 & -11.8 & $<0.001$ & -4.5 & 0.008 & -7.2 & $<0.001$ \\
\hline Serious & +1.3 & 0.257 & -3.6 & $<0.001$ & -1.8 & $<0.001$ & -1.0 & $<0.001$ \\
\hline \multicolumn{9}{|l|}{ Type of care } \\
\hline Inpatient & +4.8 & 0.351 & -0.9 & 0.808 & -16.3 & $<0.001$ & +8.4 & $<0.001$ \\
\hline Outpatient & -4.8 & 0.351 & +0.9 & 0.808 & +16.3 & $<0.001$ & -8.4 & $<0.001$ \\
\hline
\end{tabular}

to 2012, contributing factors of which were the increased CAC service availability, introduction of medication methods and provider trainings at all levels of the healthcare system. Trends in abortion-related services show a significant decrease in treatment of incomplete abortion, inverting the relationship between safe terminations and treatment of incomplete abortions as a percentage of total abortions. This is an important trend as many of the incomplete abortions were likely attempts to unsafely terminate a pregnancy, thus the downward trend suggests that abortion is becoming safer among this particular population. ${ }^{9}$

MA was nearly non-existent in 2008, but increased steadily, representing $80 \%$ of total procedures in 2012. The availability of CAC and inclusion of MA likely contributed to the observed decline in the prevalence of

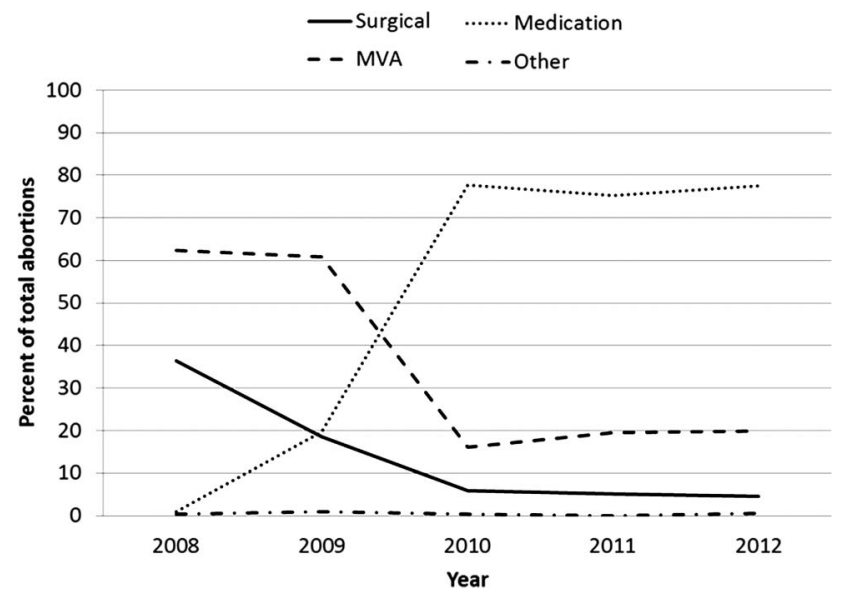

Figure 2 Trends of treatment used in abortion-related services in four tertiary hospitals in Tigray, Ethiopia (20082012). complications and a slight decline in inpatient procedures. The trends observed in the data demonstrate how increased availability of CAC services at all levels of the healthcare system can significantly reduce the burden of unsafe abortion at referral hospitals. As results from the larger project demonstrated, lower level facilities and lower level providers at hospitals took on much of the work that would have otherwise monopolised the time of physicians and clinical officers at referral facilities, thus reducing the financial and personnel burden of abortion in these hospitals. ${ }^{27}$

Results indicate that the absolute number of abortionrelated services provided increased over the period of interest. This could be explained by a likely increase in knowledge among providers regarding the change in legal status of abortion, resulting in better reporting in addition

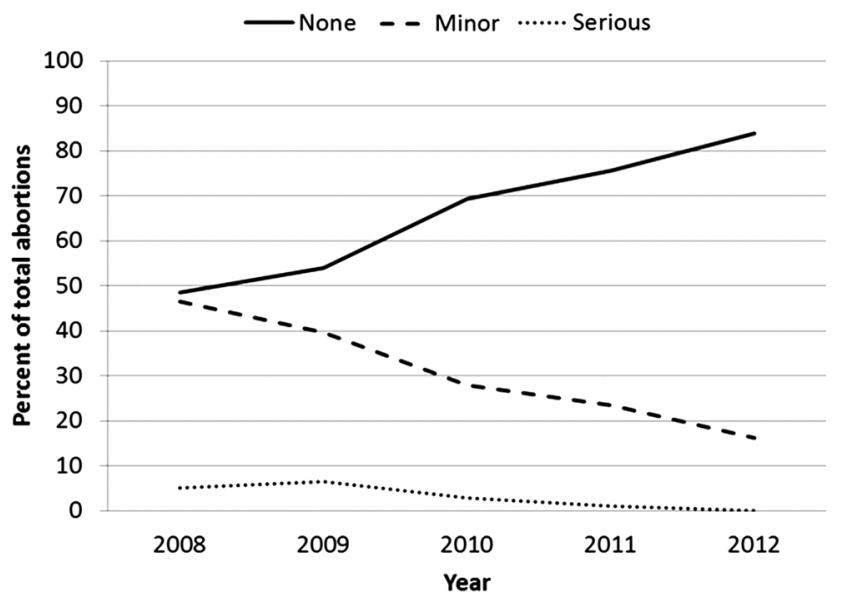

Figure 3 Trends in degree of complications in abortion-related services in four tertiary hospitals in Tigray, Ethiopia (2008-2012). 


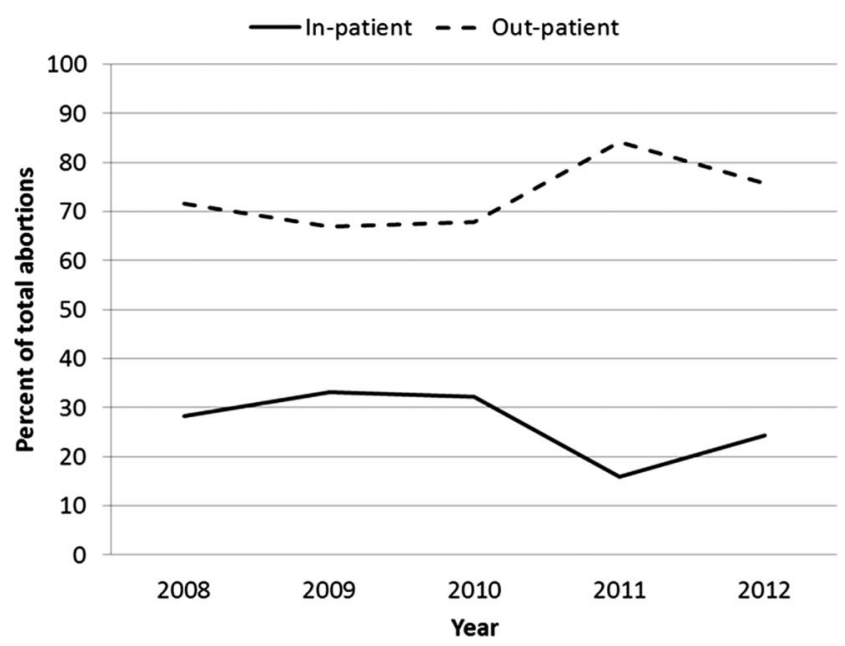

Figure 4 Trends in type of care provided for abortion-related services in four tertiary hospitals in Tigray, Ethiopia (2008-2012).

to new reporting procedures implemented by the TRHB and its partners. It is also likely that increased knowledge among women concerning abortion's legality lead more of them to seek facility based care. Nevertheless, the changes in the type of service (safe termination vs treatment of incomplete), the method (MA vs surgical) and the level of complications means a decreased burden on tertiary level facilities despite the changes in absolute number of procedures found in this study. Additionally, the training of providers at lower levels in aspects of CAC likely reduced the absolute number of women being referred for abortion-related services, further reducing the burden of unsafe abortion on referral hospitals. In particular, the availability of safe termination services at facilities closest to women (ie, health posts) likely meant less women interfering with their pregnancy themselves, which would have contributed to the reduction in treatment of incomplete abortion at the hospitals in this study. The lack of trend regarding inpatient treatment could be a result of more women receiving treatment from lower level facilities and more of the women who experience complications or who have an advanced gestational age receiving treatment at the tertiary level.

Our findings provide evidence that the utilisation and safety of abortion-related services is increasing in Tigray, but other findings looking at hospital-level data shortly after the change in the penal code had more mixed results. ${ }^{7}$ Although the AMMRs revealed a non-statistically significant downward trend over the 5-year period surrounding the abortion law revision (2003-2007), the severity of the abortion complications and the case fatality rate of abortion increased. ${ }^{7}$ Perhaps this study took place too close to the penal code changes to observe significant improvements or perhaps more women felt comfortable presenting at a facility after experiencing complications; this could also explain the lack of a significant trend in our data with regard to inpatients versus outpatients. Recent work carried out in this area achieved more consistently promising results. ${ }^{26}$ The SAC model-which is comprised of three elements that will contribute to maternal mortality reductions, including safe-induced abortion for all legal indications, treatment of abortion complications and provision of postabortion contraception-was tested in Tigray, Ethiopia from 2007 to 2009 following the change in the penal code. ${ }^{26}{ }^{29}$ Researchers saw significant increases in the availability of SAC services, the proportion of procedures that were induced abortions, the proportion of procedures that utilised recommended technologies after implementation and the proportion of patients who received modern contraceptives postabortion. ${ }^{26}$ It is apparent from these findings and ours that great strides can be made if a concerted effort is made to increase the availability of quality CAC services. Additional work in this area is required to further reduce the burden of unsafe abortion in Tigray, Ethiopia.

Availability of CAC services at all levels of the healthcare system, in particular the inclusion of medication methods, can increase access to safe abortion services, thereby reducing the financial, personnel, maternal and societal burden of unsafe abortion. MA provision requires minimal training and no equipment is needed for its use. In addition, results from studies have demonstrated that women often choose MA in lieu of other options and report high satisfaction with the method. ${ }^{16-18} \quad 30 \quad 31$ Misoprostol abortion-related services are also easy to administer, nonsurgical methods that are inexpensive and that could be provided by lower level providers for first trimester terminations. MA using the misoprostol-mifepristone combination is more efficacious than misoprostol alone and should continue to be used as the gold standard. However, for the time being, misoprostol only abortion services enable HEWs at health posts to alleviate some of the abortionrelated burden on higher level facilities, given that misoprostol tablets are available at health posts for postpartum haemorrhage. Providing services at health posts, which are closer to where clients live, would contribute to improved access, improved awareness of CAC service availability, and reduced delays in reaching care, thus potentially reducing the need for second trimester terminations.

\section{Limitations}

Data quality was an important limitation. Although we believe the hospitals' log books captured nearly complete data on the total number of abortion-related services provided (ie, we believe there is a log book entry for nearly all clients), many indicators were not filled out consistently, resulting in missing data. As a result, we used proportions in our calculations where the denominator for each indicator did not include missing responses. We assumed the proportions would not be systematically differently if the hospital staff had filled in all the information for each client, thus the missing data did not bias our findings under the assumption that it is missing at random.

Ideally, we would have been able to collect additional information regarding the percentage of total clients 
presenting at the hospital for abortion-related services from all obstetric services provided each year. Abortion-related maternal mortality is another indicator that would have been interesting to examine, but there were very few abortion deaths recorded (6 over the time period under investigation) thus the power was limited to make significant comparisons. The low number of reported abortion deaths is likely due to the fact that many women who die of abortion-related complications do not present at facilities for treatment. In addition, given the intervention trained providers at all levels of the healthcare system, capturing which patients were referrals would have given an idea of how the whole system is functioning in terms of patient flow from different levels of the healthcare system.

Despite these limitations, the data provide insights regarding recent trends in hospital-level abortion-related indicators in Tigray, Ethiopia. The trends observed in the data demonstrate how increased availability of CAC services at all levels of the healthcare system can contribute to significant reductions in the burden of unsafe abortion at referral hospitals as the number of clients served continues to increase. With 850 facilities throughout Tigray, and the efforts discussed in this study covering less than $10 \%$ of these facilities, there is still much more work to be carried out in order to further reduce the burden of unsafe abortion, but as seen in the data presented from the four hospitals that participated in the CAC pilot project, improvements are possible.

Acknowledgements We would like to acknowledge the staff and management at participating hospitals; they are doing tremendous work and their help and hard work was invaluable. We would also like to thank Melody Liu with VSI for helping prepare the data extraction form, and Asmelash Kahsay, Laura Harris and Kristina Kastler for assisting in the data extraction.

Contributors NP was the primary investigator for this project. She contributed to the data analysis and writing of the manuscript. SB helped collect the data, conducted the data analysis and helped write the manuscript. AG facilitated data collection in-country and assisted in the writing of the manuscript. All authors have read and approved the final manuscript.

Funding Funding for this project was made possible by the Bixby Center for Population, Health and Sustainability at the University of California, Berkeley.

Competing interests None.

Provenance and peer review Not commissioned; externally peer reviewed.

Data sharing statement Statistical code and data available from the corresponding author at Dryad repository, who will provide a permanent, citable and open access home for the dataset.

\section{REFERENCES}

1. World Health Organization (WHO). The global burden of disease: 2004 update. Geneva: World Health Organization (WHO), 2008.

2. World Health Organization (WHO), UNICEF, UNFPA, the World Bank. Maternal mortality in 2005: estimates developed by WHO, UNICEF, UNFPA, and the World Bank. Geneva: World Health Organization (WHO), 2007.

3. World Health Organization (WHO). Unsafe abortion: global and regional estimates of the incidence of unsafe abortion and associated mortality in 2008. 6th edn. Geneva: World Health Organization (WHO), 2011.

4. Gorrette N, Nabukera S, Salihu HM. The abortion paradox in Uganda: fertility regulator or cause of maternal mortality. $J$ Obstet Gynaecol 2005;25:776-80.
5. Sedgh G, Henshaw S, Singh S, et al. Induced abortion: estimated rates and trends worldwide. Lancet 2007;370:1338-45.

6. Singh S, Fetters $\mathrm{T}$, Gebreselassie $\mathrm{H}$, et al. The estimated incidence of induced abortion in Ethiopia, 2008. Int Perspect Sex Reprod Health 2010;36:16-25.

7. Gebrehiwot Y, Liabsuetrakul T. Trends of abortion complications in a transition of abortion law revisions in Ethiopia. $J$ Public Health (Oxf) 2009;31:81-7.

8. World Health Organization (WHO). Safe abortion: technical and policy guidance for health systems. 2nd edn. Geneva: World Health Organization (WHO), 2012.

9. Gebreselassie H, Fetters T, Singh S, et al. Caring for women with abortion complications in Ethiopia: national estimates and future implications. Int Perspect Sex Reprod Health 2010;36:6-15.

10. Abdella A, Fetters T, Benson J, et al. Meeting the need for safe abortion care in Ethiopia: results of a national assessment in 2008. Global Public Health 2013:8:417-34.

11. Gessessew A. Abortion and unwanted pregnancy in Adigrat Zonal Hospital, Tigray, north Ethiopia. Afr J Reprod Health 2010; 14:183-8.

12. Vlassoff M, Fetters $T$, Kumbi S, et al. The health system cost of postabortion care in Ethiopia. Int J Gynaecol Obstet 2012;118(Suppl 2):S127-33.

13. Faundes A, Fiala C, Tang OS, et al. Misoprostol for the termination of pregnancy up to 12 completed weeks of pregnancy. Int $J$ Gynaecol Obstet 2007;99(Suppl 2):S172-7.

14. von Hertzen $\mathrm{H}$, Piaggio G, Huong NT, et al. Efficacy of two intervals and two routes of administration of misoprostol for termination of early pregnancy: a randomised controlled equivalence trial. Lancet 2007;369:1938-46.

15. Tang OS, Miao BY, Lee SW, et al. Pilot study on the use of repeated doses of sublingual misoprostol in termination of pregnancy up to 12 weeks gestation: efficacy and acceptability. Hum Reprod 2002;17:654-8.

16. Bique C, Usta M, Debora B, et al. Comparison of misoprostol and manual vacuum aspiration for the treatment of incomplete abortion Int J Gynaecol Obstet 2007;98:222-6.

17. Dao B, Blum J, Thieba B, et al. Is misoprostol a safe, effective and acceptable alternative to manual vacuum aspiration for postabortion care? Results from a randomised trial in Burkina Faso, West Africa. BJOG 2007;114:1368-75.

18. Shwekerela B, Kalumuna R, Kipingili R, et al. Misoprostol for treatment of incomplete abortion at the regional hospital level: results from Tanzania. BJOG 2007;114:1363-7.

19. Weeks A, Alia G, Blum J, et al. A randomized trial of misoprostol compared with manual vacuum aspiration for incomplete abortion. Obstet Gynecol 2005;106:540-7.

20. Warriner IK, Wang D, Huong NT, et al. Can midlevel health-care providers administer early medical abortion as safely and effectively as doctors? A randomised controlled equivalence trial in Nepal. Lancet 2011;377:1155-61.

21. Yarnall J, Swica $Y$, Winikoff B. Non-physician clinicians can safely provide first trimester medical abortion. Reprod Health Matters 2009;17:61-9.

22. Berer M. Provision of abortion by mid-level providers: international policy, practice and perspectives. Bull World Health Organ 2009;87:58-63.

23. Central Statistical Agency, ICF Macro. Ethiopia Demographic and Health Survey 2011: Preliminary Report. Calverton, 2011.

24. Federal Democratic Republic of Ethiopia. The criminal code of the Federal Democratic Republic of Ethiopia, Proclamation No. 414/ 2004, 2005.

25. Ethiopian Ministry of Health. Technical and procedural guidelines for safe abortion care services in Ethiopia. Addis Ababa: Ministry of Health $(\mathrm{MOH}), 2006$.

26. Otsea K, Benson J, Alemayehu T, et al. Testing the safe abortion care model in Ethiopia to monitor service availability, use, and quality. Int J Gynaecol Obstet 2011;115:316-21.

27. Prata N, Gessessew A, Holston M, et al. Comprehensive abortion care pilot project in Tigray, Ethiopia: final report. Berkeley: Venture Strategies Innovations (VSI), 2011.

28. StataCorp. Stata survey data reference manual: release 11. College Station, TX: StataCorp LP, 2009.

29. Healy J, Otsea K, Benson J. Counting abortions so that abortion counts: indicators for monitoring the availability and use of abortion care services. Int J Gynaecol Obstet 2006;95:209-20.

30. Akin A, Kocoglu GO, Akin L. Study supports the introduction of early medical abortion in Turkey. Reprod Health Matters 2005; 13:101-9.

31. Winikoff B. Acceptability of medical abortion in early pregnancy. Fam Plann Perspect 1995;27:142-8, 85. 